University of Nebraska - Lincoln

DigitalCommons@University of Nebraska - Lincoln

November 1993

\title{
Piezomodulated-Raman-spectroscopy study of the phase transition in phenothiazine
}

\author{
J. Sartwell \\ University of Nebraska - Lincoln \\ Craig J. Eckhardt \\ University of Nebraska - Lincoln, ceckhardt1@unl.edu
}

Follow this and additional works at: https://digitalcommons.unl.edu/chemistryeckhardt

Part of the Chemistry Commons

Sartwell, J. and Eckhardt, Craig J., "Piezomodulated-Raman-spectroscopy study of the phase transition in phenothiazine" (1993). Craig J. Eckhardt Publications. 15.

https://digitalcommons.unl.edu/chemistryeckhardt/15

This Article is brought to you for free and open access by the Published Research - Department of Chemistry at DigitalCommons@University of Nebraska - Lincoln. It has been accepted for inclusion in Craig J. Eckhardt Publications by an authorized administrator of DigitalCommons@University of Nebraska - Lincoln. 


\title{
Piezomodulated-Raman-spectroscopy study of the phase transition in phenothiazine
}

\author{
J. Sartwell* and C. J. Eckhardt ${ }^{\dagger}$ \\ Department of Chemistry, University of Nebraska, Lincoln, Nebraska 68588-0304
}

(Received 14 June 1993)

\begin{abstract}
Single crystals of phenothiazine undergo a displacive order-disorder phase transition near $250 \mathrm{~K}$. The microscopic mechanism of this transition is not clear owing to the complexity of a disordered lattice and the lack of direct mode-anharmonicity data. Unstable optical modes have been observed in phenothiazine and are regarded as coupled, by symmetry, to an acoustic mode that becomes overdamped through the phase transition. The latter is considered to have the primary role in the phase transition. This premise is in doubt, however, based on the optical-mode anharmonicity directly observed by uniaxialpiezomodulation-Raman-spectroscopy results presented here. Anharmonic responses are observed in the low-temperature spectra at frequencies that correspond to room-temperature Raman values and thus support persistent metastable, room-temperature, lattice structures that are intergrown within the lowtemperature phase. This result is indicative of a first-order phase change. A $22-\mathrm{cm}^{-1}$ mode at room temperature displays a signficantly anharmonic response that may correspond to the primary order parameter or to coupling with an overdamped infrared active mode.
\end{abstract}

\section{INTRODUCTION}

Single crystals of phenothiazine undergo a displacive, order-disorder, phase transition that results in the formation of visible, lamellar striae on the crystal surface. The phase transition was initially hypothesized by Nakayama et al. ${ }^{1}$ to be ferroelastic. Temperature-dependent, longwavelength, acoustic vibrational modes have been observed. ${ }^{2}$ In a previous paper, however, it was shown by Brillouin scattering that the high-symmetry acoustic modes do not play a significant role in the structural transition and the crystal was suggested to be ferrobielastic. ${ }^{3}$ The observed striae occur as a result of molecular ordering within large, polysynthetically twinned, domains that are also present at room temperature but with boundaries blurred by disorder.

The low-energy, Raman-active, lattice modes display a significant amount of anomalous behavior under changes in temperature and pressure. ${ }^{4,5}$ One mode, a $B_{2 g}$ mode at $15 \mathrm{~cm}^{-1}$, red shifts $20 \mathrm{~cm}^{-1}$ upon cooling to $90 \mathrm{~K}$. In the NMR study of Sircar, Mishra, and Gupta, ${ }^{6}$ one lowenergy optical lattice mode was discovered to have a significant vibrational amplitude that dampens dramatically upon cooling. In addition, recent NMR studies point to a phase transition that is governed by strong rotation-translation coupling rather than simply by an over-damping of an acoustic mode. ${ }^{7}$ Nakayama, Ishii, and Sawada ${ }^{8}$ and Ecolivet et al. $^{2}$ have determined, using the order parameter model of Landau, that the temperature-sensitive optical modes play only a secondary role in the phase transition. However, their conclusions are based on primary strain instabilities and are open to question.

Additional interest in the optical modes of phenothiazine has been sparked by the speculated existence of a low-energy molecular vibrational (butterfly) mode which may play a role in the phase transition. ${ }^{4} \mathrm{~A}$ major reason for the study of molecules like phenothiazine is to investi- gate the interaction between molecular and lattice potentials in influencing a phase transition.

The phase transition of phenothiazine itself represents a severe departure from a harmonic lattice. ${ }^{9}$ Mode anharmonicity is difficult to measure, is usually assessed by indirect methods, and is consequently hard to model or evaluate. The technique of uniaxial, piezomodulated, Raman spectroscopy (UPRS) has recently been shown, both theoretically ${ }^{10}$ and experimentally, ${ }^{11-13}$ to provide a direct probe of optical-mode anharmonicity through the coupling of two optical modes with a periodic, uniaxial stress. In this paper we report the results of UPRS on single crystals of phenothiazine. The Raman-active modes between $10-110 \mathrm{~cm}^{-1}$ were examined by this method at room temperature and at 90 or $100 \mathrm{~K}$, in order to obtain insight into the anharmonicity of the crystal above and below the phase transition temperature.

In the next section the principles of piezomodulated Raman spectroscopy are reviewed. This is followed by a presentation of the details of the experiment and of the ensuing results. The subsequent session discusses the results and frames an inclusive explanation for the observations.

\section{PIEZOMODULATED RAMAN SPECTROSCOPY}

Phase transitions signal the presence of highly anharmonic vibrational modes within a crystal. Those modes whose associated motions are directly responsible for the structural transformation are most strongly affected. Historically, anharmonicity has been directly estimated by frequency shifts from harmonic values and bandwidth changes throughout the phase transition by the techniques of x-ray, Raman, Brillouin, and neutron scattering. ${ }^{14}$ Unfortunately, a proper evaluation of a change in frequency requires examination of pertinent combination and overtone bands that are rarely observed with lattice modes. The evaluation of subtle bandwidth changes 
through the phase transition requires complicated fitting procedures using assumed band shapes.

Piezomodulated Raman scattering is a spectroscopy that measures strain-induced frequency shifts for optical modes. ${ }^{10}$ The UPRS band shapes are derivativelike with responses that are directly proportional to the anharmonicity of the optical mode coupled to a strain generated under predetermined conditions. The coupling of select strains to anharmonic optical modes will always be present in real crystal systems. In many cases, a secondary coupling between a strain and the order parameter may also be associated with a phase transition. Thus the UPRS experiment can survey both the primary and secondary order parameters associated with the phase transition.

The key concept behind the theory is that phononphonon coupling can be mediated by an acoustic phonon $\hat{q}_{\beta}$ associated with a static strain $e_{\alpha \beta}$ that is generated within the crystal by a periodically applied uniaxial stress:

$$
e_{\alpha \beta}=\lim _{\hat{q}_{\beta} \rightarrow 0} Q_{\alpha}(\hat{q}) \hat{q}_{\beta}
$$

Here $Q_{\alpha}(\hat{q})$ are the normal coordinates of the acoustic phonon.

Experimentally, the Raman response measured synchronously with the stress modulation represents the difference between scattering responses at the peak-topeak compressions and extensions of the crystal. The Hamiltonian for a strained lattice appropriate for the Raman scattering experiment is

$$
H=H_{0}+H^{\prime} \text {, }
$$

where $H_{0}$ and $H^{\prime}$ are the potential energies of an underformed and deformed unit cell, respectively. The Hamiltonian $H^{\prime}$ generally can be quite complicated. However, simplification is achieved by considering only the experimentally accessible zone center $\hat{q} \sim 0$ optical modes which allows for renormalization of the Hamiltonian by splitting the normal coordinates of the optical modes into two parts:

$$
Q(0, j)=Q_{j}^{\mathrm{el}}+Q_{j}
$$

$Q_{j}^{\text {el }}$ denotes the elastic displacement of the molecules for a given strain and $Q_{j}$ is the instantaneous equilibrium position of this vibration and, as such, its average position is unchanged. The effective renormalized Hamiltonian of the deformed lattice becomes

$$
H^{\prime}=\frac{1}{2} v \sum_{\alpha \beta \gamma \delta} C_{\alpha \beta, \gamma \delta} e_{\alpha \beta} e_{\gamma \delta}+\frac{1}{2} \sum_{j j^{\prime}} \Omega_{j j^{\prime}} Q_{j} Q_{j^{\prime}},
$$

where the matrix $\Omega_{j j^{\prime}}$ represents the mode anharmonicity

$$
\Omega_{j j^{\prime}}=\omega_{0 j}^{2} \delta_{j j^{\prime}}+\sum_{\alpha \beta} \phi_{\alpha \beta j j^{\prime}} e_{\alpha \beta}
$$

and $\phi_{\alpha \beta j j^{\prime}}$ represents the coupling between two optical phonons $Q_{j} Q_{j}$, and includes both lattice and intramolecular modes. The eigenvectors of Eq. (5) are still harmonic phonons but no longer correspond to plane-wave solu- tions. The eigenfrequencies $\omega_{\gamma}^{2}$ will strongly depend on the anharmonic interactions between the strain-mediated, optical phonons. The magnitude of $\phi_{\alpha \beta j j^{\prime}}$ is then manifested by the differences in eigenfrequencies between the strained and unstrained crystal. An ideally harmonic mode will give no UPRS response because the corresponding coupling constant will be zero. Internal strains are considered to be of the same symmetry as the external stress, and their effects also are assumed to be minimal.

A data analysis of the UPRS signal requires an expression for the intensity of the Raman response in terms of the coupling constant $\phi_{\alpha \beta j j^{\prime}}$ and the resultant strain. In the UPRS experiment, the observed intensity is actually a function of the Raman response between both extensive and compressive states of the stress cycle. The stresses used in UPRS are small and the resulting strains are nearly infinitesimal. Because the strains are so small and within the elastic limit, the frequency shifts and the coupling constants must be determined indirectly. Luty and Eckhardt developed an expression for the intensity of a UPRS signal in terms of a strain derivative of the Raman response and the coupling constant $\phi_{\alpha \beta j j^{\prime}{ }^{10}}$ For sufficiently small strains the UPRS intensity is given by

$\Delta I(\omega)=\frac{\partial I(\omega)}{\partial e_{\alpha \beta}} \Delta e_{\alpha \beta}=-\Delta e_{\alpha \beta} \operatorname{Im}\left[\chi^{0}(\omega) \phi_{\alpha \beta} \chi^{0}(\omega)\right]$,

where $\chi^{0}$ and $\phi_{\alpha \beta}$ are the electronic susceptibility and the coupling constants, respectively, for a one phonon oscillator and $\Delta e$ is defined as

$$
\Delta e=\left(e_{\text {extension }}-e_{\text {compression }}\right)_{\mathrm{rms}} \text {. }
$$

Determination of coupling constants is accomplished through a curve-fitting procedure described by White and Eckhardt. ${ }^{13}$ By expressing the electronic susceptibility of the crystal as

$$
\chi^{0}(\omega)=\left[\omega_{0}^{2}-\omega^{2}+i \omega \gamma\right]^{-1}
$$

the unstrained Raman response can be obtained:

$$
I(\omega)=A \frac{\omega \gamma}{\left(\omega_{0}^{2}-\omega^{2}\right)^{2}+\omega^{2} \gamma^{2}},
$$

where $\gamma$ is the full width at half-height of the Raman band and $A$ scales the intensity of the band. The corresponding expression for the piezomodulated Raman response is given as

$$
\Delta I(\omega)=\frac{\partial I(\omega)}{\partial e_{\alpha \beta}} \Delta e_{\alpha \beta}=-\Delta e_{\alpha \beta} \frac{2 \phi_{\alpha \beta} A \omega \gamma\left(\omega_{0}^{2}-\omega^{2}\right)}{\left[\left(\omega_{0}^{2}-\omega^{2}\right)^{2}+\omega^{2} \gamma^{2}\right]^{2}} .
$$

The conventional Raman band is fit to Eq. (9) to obtain $\omega_{0}, \gamma$, and $A$; these values are used in Eq. (10) to obtain $\Delta e_{\alpha \beta} \phi_{\alpha \beta}$ from the UPRS response. The stress generated by the lead zirconate titanate (PZT), piezoelectric modulator is known and the resulting strain within the crystal can be calculated separately to yield $\phi_{\alpha \beta}$, if the crystal elastic constants are known.

It has been demonstrated by White and Eckhardt ${ }^{13}$ that UPRS also permits the semiempirical determination of the molecular motions associated with eigenmodes of molecular crystals. UPRS was found to provide experi- 
mental verification of the eigenmodes determined by lattice dynamical calculation. As Eq. (10) shows, the band shape of the UPRS response is derivativelike with respect to strain but not frequency. White and Eckhardt ${ }^{13}$ have shown the two types are distinctly different. The distinction is significant in terms of the phase of the derivative. The phase of a UPRS band can be either positive or negative with respect to the initial positive or negative lobe of the derivative. A positive-type band was found to correspond to an increase in the vibrational frequency under a compressional stress and a decrease in frequency under a tensile expansion. A negative-type UPRS band displayed the opposite behavior.

Uniaxial stresses along high-symmetry axes within the crystal generate, in principle, pure compression or expansion strains within the crystal. The frequencies of those modes that can couple are increased if the potential energy of the sample is increased by bringing neighboring molecules closer together. In the case of a shear strain, for example, a compression or expansion will be induced perpendicular to the direction of applied stress and $180^{\circ}$ out of phase with the periodic modulation, provided the associated elastic constants are positive. In these cases, the eigenvectors with components perpendicular to the uniaxial stress directions will exhibit the opposite action and are associated with a negative UPRS band.

Selection rules also apply to the UPRS experiment. The optical phonons that can couple are dictated by the direct product

$$
e_{\alpha \beta} Q_{j} Q_{j^{\prime}}
$$

For totally symmetric strains, anharmonic phonons of the same symmetry can couple and phonons of different symmetries can couple for nonsymmetric strains.

The coupling term $\phi_{\alpha \beta} e_{\alpha \beta} Q_{j} Q_{j^{\prime}}$ in Eq. (4) shows that if one, two, or all three of the terms, $e_{\alpha \beta}, Q_{j}$, or $Q_{j^{\prime}}$, are anharmonic, $\phi_{\alpha \beta}$ should be large relative to the strain's or mode's role in any dynamic behavior occurring within the sample. In terms of Landau theory, it should be possible to directly identify those primary and secondary elements of the phase transition by examining the temperature and pressure dependence of $\phi_{\alpha \beta}$.

\section{EXPERIMENTAL}

The phenothiazine used in this experiment was a pale yellow solid that was purchased from Aldrich Chemical Company at an approximate purity of $98 \%$. The purification and vapor-phase growth of single crystals is described in an earlier paper. ${ }^{3}$

Twinning of the phenothiazine crystals was common. At least one-third of the harvest of vapor-grown crystals were observed under a polarizing microscope to be twinned at room temperature with one region of the crystal extinguishing the reflected light at different orientations than another under crossed polarizers. Typically, macroscopic twinning of room-temperature samples did not occur in a regularly definable fashion along any of the unit cell axes or lines of symmetry. Rather, the twinning occurred as intergrowths within the larger crystal. Twin boundary lines would not readily cleave nor could they be reoriented by the application of a small stress upon the crystal edges. Room-temperature twinned crystals were not used in any experiments.

Twinning is important because all crystals of phenothiazine undergo a spontaneous twinning below $T_{c}$ and these low-temperature twins form in domainlike structures throughout the crystal. These twins are unrelated to those observed at room temperature which exhibited random intergrowth. The low-temperature twin always occurred in a lamellar fashion with domain boundaries perpendicular to the $a$ or $c$ axes $^{3}$ and may correspond to the polysynthetic twinning described by van de Waal and Feil ${ }^{15}$ or the disordered orthorhombic crystal of Stigler and Stezowski. ${ }^{16}$

The experimental Raman apparatus following the design of Tobin ${ }^{17}$ is described in detail by White. ${ }^{12}$ The original apparatus, however, had to be modified in order to incorporate a Janis Supertran-B model ST-3 lowtemperature dewar that was fitted with a piezomodulation mount. Temperatures in the range of $90-100 \mathrm{~K}$ were maintainable to within $\pm 1^{\circ}$. A complete description of the piezomodulation mount, and associated electronics for low-temperature use is described elsewhere. ${ }^{18} \mathrm{~A}$ 1-in. PZT bar was typically used and stress on the order of $2 \times 10^{-15} \mathrm{kbar}$ (peak-to-peak) rms was generated.

Single crystals of phenothiazine, prepared in the same fashion as those in the Brillouin experiment, ${ }^{3}$ were mounted in the piezomodulation cradle. Phenothiazine is an extremely fragile crystal and care must be taken not to crack the samples, especially if the direction of applied uniaxial stress is perpendicular to the cleavage plane perpendicular to the $c$ axis. $20-25 \mathrm{~h}$ of continuous periodic stress causes the crystal to fatigue and delaminate. A crystal was deemed unusable if the intensity of the conventional Raman scattering of the crystal deteriorated more than $10 \%$. Although the lamellar cleavage planes led to an eventual weakening of the crystal, the domains that formed below $T_{c}$ appeared to strengthen the sample (cleavage planes were perpendicular to the domain boundaries). Crystal cracking typically occurred only at room temperature or after return to room temperature.

\section{RESULTS: UPRS AND PHENOTHIAZINE}

The habit and morphology of the phenothiazine crystal dictated the directions along which the uniaxial stress was applied because the fragility of the crystal prevented cutting of specific faces. All stress and scattering configurations were determined in a fashion that allowed normal incidence to a crystal face and observation of normal scattering in a $90^{\circ}$ scattering geometry imposed by the instrumental constraints. A typical crystal mounting is displayed in Fig. 1, showing the generation of an $a b$ shear and a small flexure.

A direct $a c$ or $b c$ shear strain, which would give rise to an $e_{5}$ strain, could not be generated because the crystals of phenothiazine were too thin for effective stress modulation in the necessary geometry. However, because the most compressible direction within the crystal is parallel to the $c$ axis, the crystal tended to bend and delaminate in the plane perpendicular to this axis. This flexure of the 


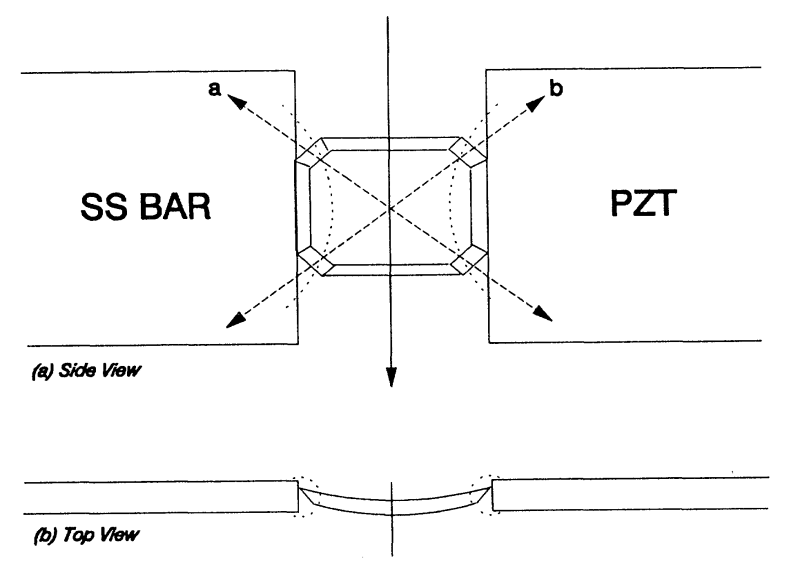

FIG. 1. Crystal in piezomount.

crystal may unavoidably provide an $e_{5}$ strain in all modulation configurations except for uniaxial stresses parallel to the $c$ axis. Due to the scattering configuration imposed by the stress geometries, most of the spectra were of mixed symmetry character.

Figures 2-7 show the conventional Raman and the piezomodulated Raman spectra at 295 and $90 \mathrm{~K}$ for the various types of applied uniaxial stresses and polarization symmetries. Notation for the Raman-scattering configuration is that of Porto, Giordmaine, and Damen. ${ }^{19}$ The atypical designation $a \Varangle b$ refers to a direction that bisects the $a$ and $b$ axes (see Fig. 1).

\section{DISCUSSION}

The study of phenothiazine presents a severe test of the UPRS technique for several reasons: (1) The crystal structure of either phase is still in doubt and the system is probably disordered; (2) phenothiazine forms structural domains and domain walls; (3) phenothiazine is very compliant along one axis, to the point that it cleaves readily; (4) the eigenvectors of motion are complex because the molecules do not lie at centers of inversion in any of the proposed structures; (5) the UPRS responses are not completely unambiguous because phenothiazine could not be cut and the symmetry of an optical mode could not be selected. Nevertheless, a number of significant interpretations can be made concerning the phase transition in phenothiazine as well as for piezomodulated Raman spectroscopy in general.

In order to determine the coupling constants associated with the experimental stresses, the nature of the corresponding generated strain must be determined first. Under a given uniaxial stress the strains produced can be determined by the relation

$$
e_{\alpha \beta}=C_{\alpha \beta \gamma \delta}^{-1} \sigma_{\gamma \delta}=S_{\alpha \beta \gamma \delta} \sigma_{\gamma \delta},
$$

where $S_{\alpha \beta \gamma \delta}$ is the compliance tensor. The compliance tensors at 295 and $225 \mathrm{~K}$ were calculated from the elastic constant data obtained from Brillouin data. ${ }^{3}$ The type of strains generated by [100], [010], and [001] uniaxial stresses are essentially equivalent, respectively, at high and low temperatures. The [110] shear stress generates, in principle, only $e_{4}$ and $e_{6}$ strains and the UPRS response should be independent of the response from the compresşional stresses. Nonetheless, a large, nonsymmetric, strain along the $c$ axis will always predominate for all these stress directions because of the very high compressibility of the phenothiazine crystal along $c$. Thus, the UPRS response for all the stresses should be similar thereby making an evaluation of the eigenvectors of motion for each mode impossible. The off-diagonal elements of the compliance tensors for phenothiazine determined for the three different structures are in some cases larger than the diagonal elements. Therefore, the actual anharmonicity must be expressed as the sum $\phi_{\alpha \beta j j^{\prime}} e_{\alpha \beta}$ (Einstein summation convention assumed).

The Raman and piezomodulated Raman responses of the optical modes at room temperature were both very sensitive to disorder and domains. At room temperature, many of the Raman bands displayed broad band shapes. This is consistent with a great deal of orientational change or disorder. In many cases, the bands also appeared as clusters of many broad peaks with slightly different but distinct frequencies and is consistent with twinning or domain formation above $T_{c}$ previously deduced from Brillouin data. ${ }^{3}$ The UPRS response of the room-temperature crystals was also suggestive of this by showing a number of closely spaced, derivativelike, responses in the frequency range $10-15 \mathrm{~cm}^{-1}$ (see Figs. 2 and 3). In fact, the fitting of the UPRS spectra to Eq. (10)

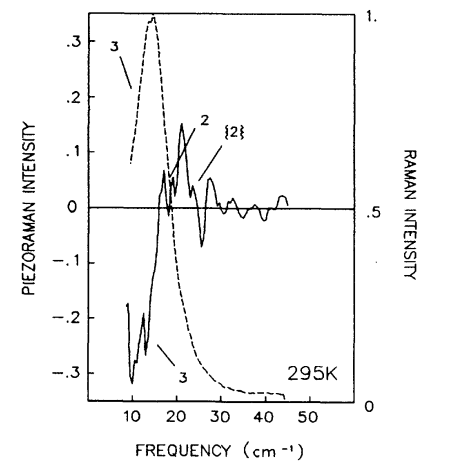

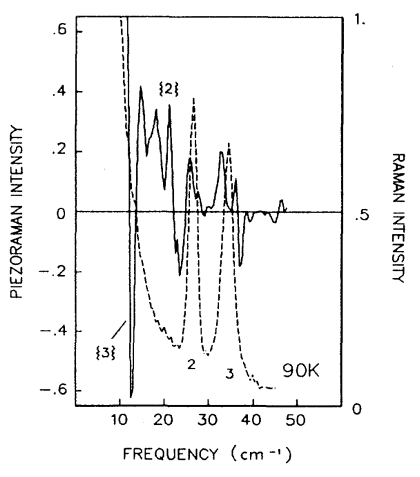

FIG. 2. Piezomodulated Raman spectra. Stress: $a b$ shear. Scattering configuration: $a \Varangle b(c a) c$ at 90 and 295 K. UPRS (-) Raman (---). Numbers indicate mode assignments in Tables III and IV. 

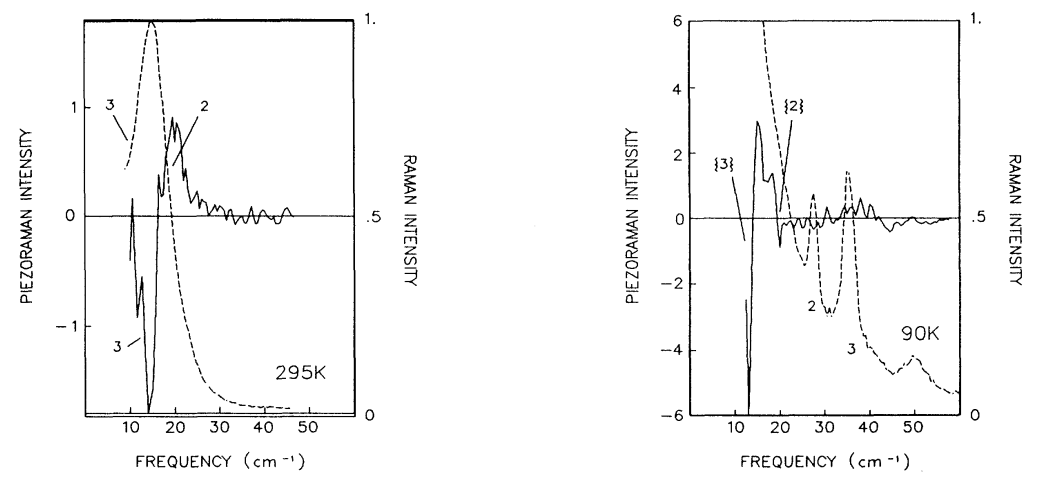

FIG. 3. Piezomodulated Raman spectra. Stress: $b$ axis. Scattering configuration: $a(c a) c$ at 90 and $295 \mathrm{~K}$.

in these cases could only be accomplished by assuming a set of closely spaced anharmonic modes. These "multiplets" are attributed to separate responses of particular domains in the lattice.

A significant feature of the UPRS spectra of the "multiplets" at room temperature is that the relative phases of the responses often differ under the same applied stress. This occurs with all stress configurations. This is attributed to strain pinning around a domain wall as illustrated in Fig. 8. The domain walls form in order to lower the free energy of the crystal, and are considered to be free, or nearly free, of stress. Anharmonicity, however, at a domain wall or pinning site clearly will be different or $180^{\circ}$ out of phase with the area away from the domain boundary if a mode is affected by the domain structures. Of course, domain pinning and fluctuations complicate interpretation of the phases of the UPRS responses when assigning the character of the lattice or molecular motion since no clear phase pattern can be established.

The determination of $\phi_{\alpha \beta j j^{\prime}} e_{\alpha \beta}$ requires a prior fit to the corresponding Raman spectra. In numerous instances distinct Raman band shapes were not observed. In some cases, large responses were observed with UPRS for Ra-

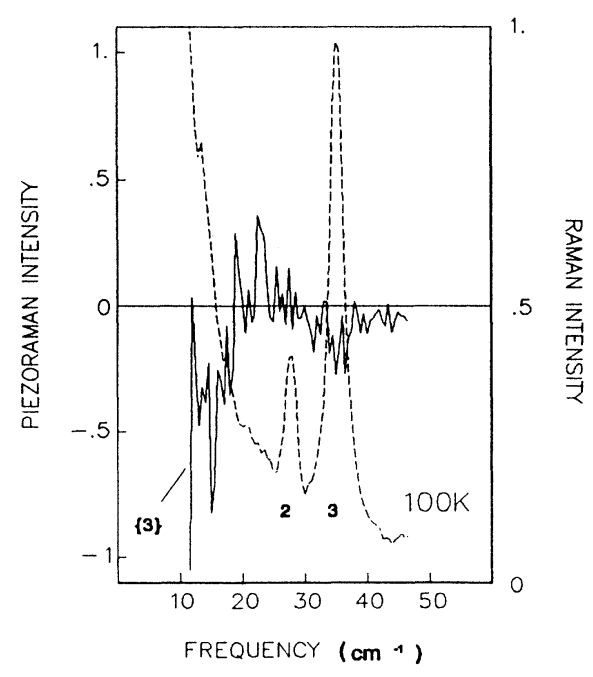

FIG. 4. Piezomodulated Raman spectra. Stress: $a$ axis. Scattering configuration: $b(c a) c$ at $100 \mathrm{~K}$. man bands that displayed little or no intensity. A UPRS response can occur when metastable regions exist in the lattice. ${ }^{11}$ In addition, several of the Raman bands overlap at the same frequency. The UPRS experiment was able to resolve individual modes better than conventional Raman techniques but it is difficult to determine the band shapes and linewidths of individual Raman modes or UPRS responses. Because of these limitations, the very broad Raman band shapes observed in this experiment were fit with estimates of the linewidths and intensities of individual Raman modes determined from the spectra taken by Nakayama and $\mathrm{Ishii}^{4}$ for approximately the same scattering geometry. The fitting procedure involved a weighted minimization of the Lorentz equation for a Raman band shape. The calculated coupling constants are given in Tables I and II.

The optical-mode, strain-dependent, anharmonic coupling constants $\phi_{\alpha \beta j j^{\prime}} e_{\alpha \beta}$, determined from the spectra are treated for each mode pair (same mode at 295 and $90 \mathrm{~K}$ or $100 \mathrm{~K}$ ) for the different stress configurations for which the pair was observed. The discussion will begin with the mode of lowest frequency. Modes are numbered using the designations of Nakayama and Ishii ${ }^{4}$ whose system was based on the low-temperature spectra and is presented in Tables III and IV. Modes determined by the lattice dynamic calculation of Criado $^{20}$ are also given in Table IV. Modes not presented either did not display a UPRS signal that could be distinguished from the background noise, or were not observable in the scattering geometries dictated by the experiment.

Previous mode assignments have been made by Nakayama and Ishii ${ }^{4}$ from Raman scattering and by Criado ${ }^{20}$ using lattice dynamical calculations assuming a $P 2_{I} / n\left(C_{2 h}\right)$ low-temperature structure.

The conventional Raman bands shifted in frequency between $5-20 \mathrm{~cm}^{-1}$ at low temperature. In addition, their full width at half maximum (FWHM) significantly decreased at $90 \mathrm{~K}$. The narrowing of the Raman band shapes is consistent with an order-disorder structural phase transition. Most of the conventional Raman bands, however, were also strongly affected by the presence of domains, or twinning, or both. Domain pinning may become especially significant at low temperature because the phenothiazine crystal was always under a static, uniaxial, compression due to the thermal contraction of the brass piezomodulation mount. 

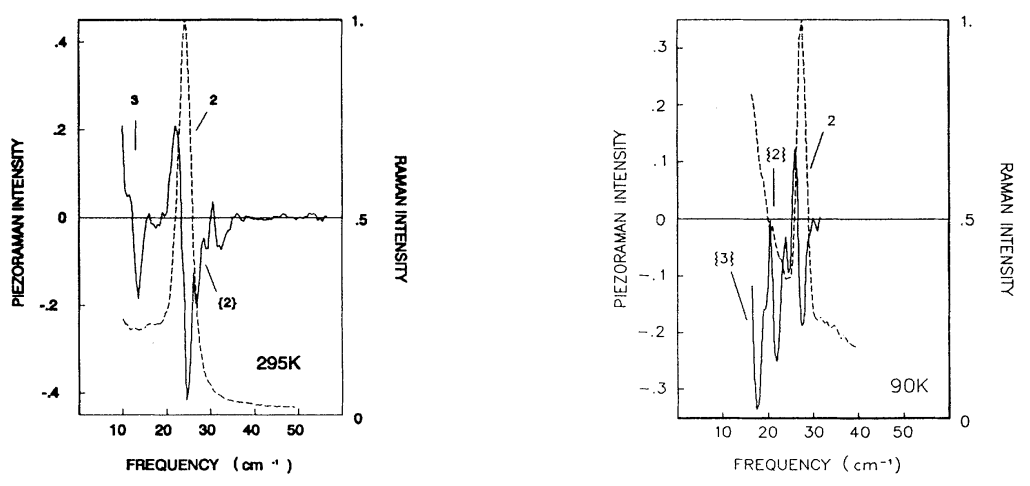

FIG. 5. Piezomodulated Raman spectra. Stress: $c$ axis. Scattering configuration: $a \Varangle b(\{a \Varangle b\} c) a \Varangle b$ at 90 and $295 \mathrm{~K}$.
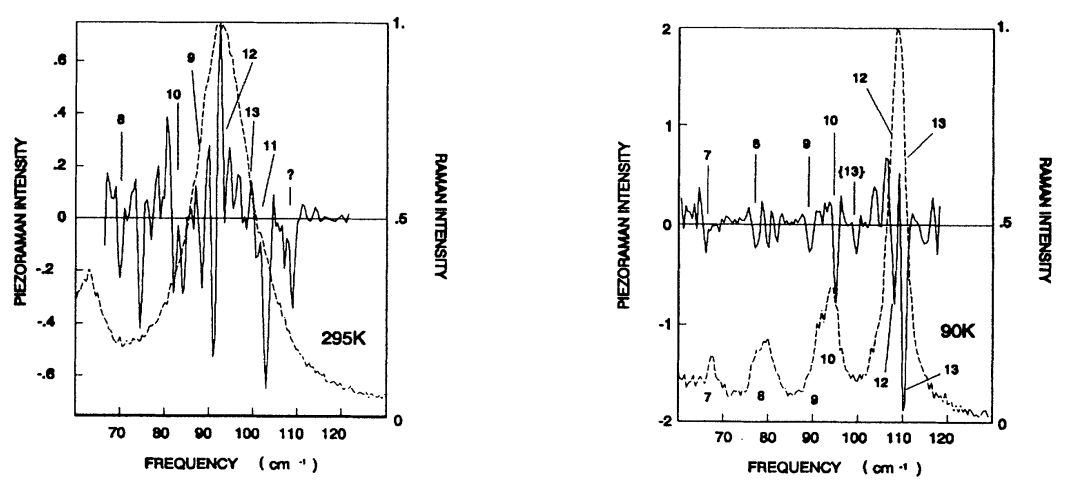

FIG. 6. Piezomodulated Raman spectra. Stress: $a b$ shear. Scattering configuration: $a \Varangle b(\{a \Varangle b\} c) a \Varangle b$ at 90 and $295 \mathrm{~K}$.
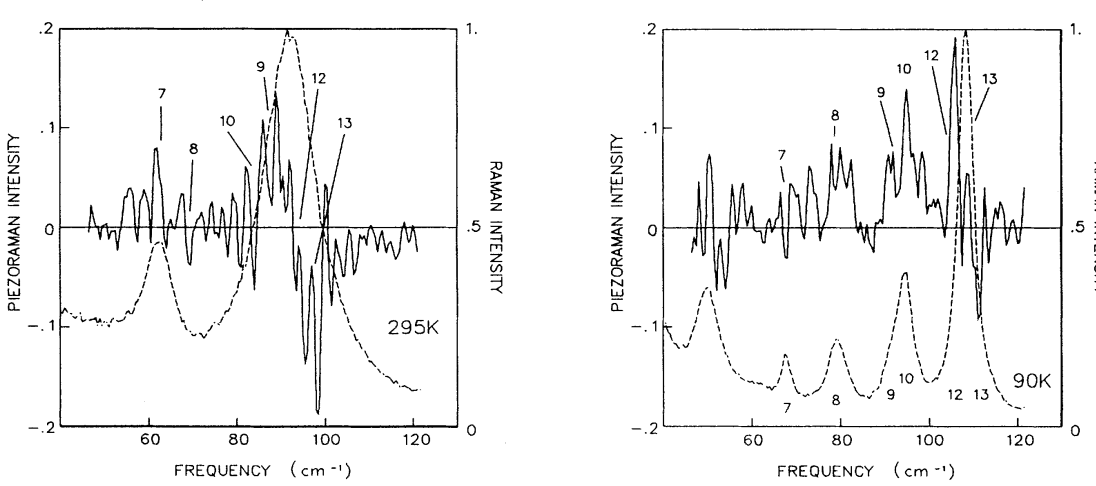

FIG. 7. Piezomodulated Raman spectra. Stress: $a$ axis. Scattering configuration: $b(a a) c$ at 90 and $295 \mathrm{~K}$.

TABLE I. Coupling constants $\phi_{\alpha \beta} \epsilon_{\alpha \beta}\left(\mathrm{cm}^{-1}\right)$ for specified uniaxial stresses (in brackets) for modes in the frequency range $10-40 \mathrm{~cm}^{-1}$. Braces indicate a mode present under metastable conditions or mode coupling to an IR-active mode (see text).

\begin{tabular}{|c|c|c|c|c|c|c|c|}
\hline $\begin{array}{l}\text { Mode } \\
\mathrm{cm}^{-1}\end{array}$ & $\begin{array}{c}{[100]} \\
100 \mathrm{~K} \\
\end{array}$ & $\begin{array}{c}{[010]} \\
295 \mathrm{~K} \\
\end{array}$ & $\begin{array}{c}{[010]} \\
100 \mathrm{~K} \\
\end{array}$ & $\begin{array}{c}{[001]} \\
295 \mathrm{~K}\end{array}$ & $\begin{array}{c}{[001]} \\
100 \mathrm{~K}\end{array}$ & $\begin{array}{c}{[110]} \\
295 \mathrm{~K} \\
\end{array}$ & $\begin{array}{c}{[110]} \\
100 \mathrm{~K} \\
\end{array}$ \\
\hline $10.9(3 H)$ & $\{-390\}$ & 50 & $\{-1680\}$ & & & -26 & -72 \\
\hline 12.5 & & & & -238 & & 3 & 50 \\
\hline $14.0(3 H)$ & $\{370\}$ & -63 & & & & & \\
\hline $15.9(3 H)$ & $\{-190\}$ & & $\{1400\}$ & 113 & & 5 & \\
\hline $17.5(3 H)$ & & 85 & $\{-520\}$ & -44 & $\{-200\}$ & -6 & -50 \\
\hline $34.5(3 L)$ & -30 & & -6 & & & & -9 \\
\hline $22.5(2 H)$ & $\{-170\}$ & -5 & & -4 & $\{-34\}$ & -7 & $\{-130\}$ \\
\hline $27.0(2 L)$ & -50 & & -1 & $\{51\}$ & -1 & & -1 \\
\hline
\end{tabular}




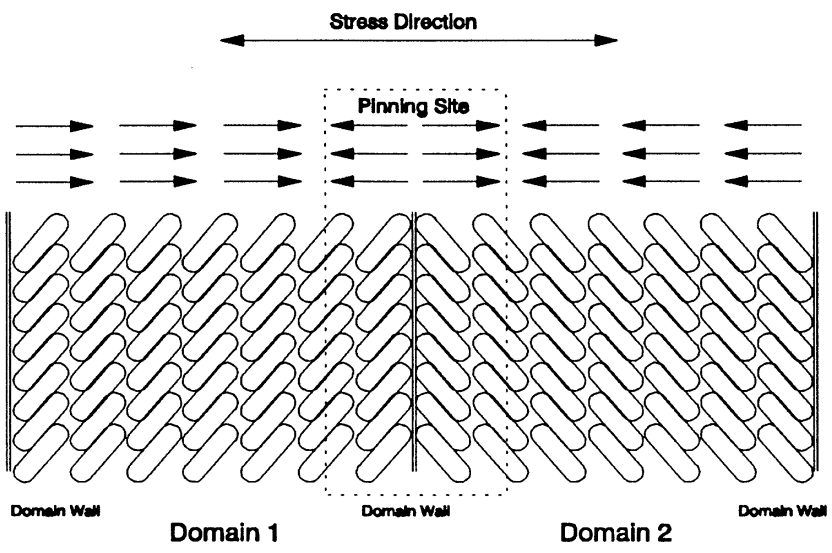

FIG. 8. Strain pinning at a domain wall under a uniaxial stress.

A striking feature of the UPRS responses is that large anharmonic signals were recorded from the lowtemperature phase $(90-100 \mathrm{~K})$ of phenothiazine in the frequency range $10-17 \mathrm{~cm}^{-1}$. This is significant because mode $3\left[14.7 \mathrm{~cm}^{-1}(295 \mathrm{~K}) \rightarrow 34.3 \mathrm{~cm}^{-1}(90 \mathrm{~K})\right]$ was observed to shift in frequency to $34.3 \mathrm{~cm}^{-1}$ in the lowtemperature phase but no UPRS signal was expected in the lower-frequency range. Anharmonic coupling constants of these low-temperature UPRS responses below a frequency of $20 \mathrm{~cm}^{-1}$ ranged from -190 to $1680 \mathrm{~cm}^{-1}$ and were the largest values observed overall. The average value for the coupling constants of all modes sampled was less than $130 \mathrm{~cm}^{-1}$. The corresponding lowtemperature conventional Raman band in the frequency range $10-17 \mathrm{~cm}^{-1}$ was of very low intensity and, in some configurations, not even observed. There are two possible explanations for this anomalous behavior: the existence

TABLE II. Coupling constants $\phi_{\alpha \beta} \epsilon_{\alpha \beta}\left(\mathrm{cm}^{-1}\right)$ for specified uniaxial stresses (in brackets) for modes in the frequency range $60-120 \mathrm{~cm}^{-1}$. Braces indicate a mode present under metastable conditions or mode coupling to an IR-active mode (see text).

\begin{tabular}{lcccc}
\hline \hline \multicolumn{1}{c}{$\begin{array}{c}\text { Mode } \\
\mathrm{cm}^{-1}\end{array}$} & $\begin{array}{c}{[100]} \\
295 \mathrm{~K}\end{array}$ & $\begin{array}{c}{[100]} \\
100 \mathrm{~K}\end{array}$ & $\begin{array}{c}{[110]} \\
295 \mathrm{~K}\end{array}$ & $\begin{array}{c}{[110]} \\
100 \mathrm{~K}\end{array}$ \\
\hline $68.0(8 H)$ & -77 & & -81 & \\
$79.0(8 L)$ & & 2 & & 31 \\
$84.0(10 H)$ & 99 & & 110 & \\
$94.5(10 L)$ & & -25 & & -103 \\
$87.9(9 H)$ & 36 & & 5 & \\
$91.0(9 L)$ & & 4 & & 124 \\
$91.0(12 H)$ & -49 & & 123 & \\
$108.5(12 L)$ & & -26 & & -57 \\
$97.0(13 H)$ & 110 & $\{7\}$ & & 59 \\
$109.5(13 L)$ & & & & $\{114\}$ \\
$100.0(11 L)$ & & & & $\{-424\}$ \\
$73.0(?)$ & & & 62 & -25 \\
$112.5(?)$ & & & & \\
$118.0(?)$ & & & & \\
\hline \hline
\end{tabular}

TABLE III. Experimental and calculated lattice frequencies (in $\mathrm{cm}^{-1}$ ) of the low-temperature monoclinic phase of phenothiazine.

\begin{tabular}{|c|c|c|c|}
\hline Mode & Symmetry & $\begin{array}{c}\text { Nakayama and Ishii } \\
90 \mathrm{~K}\end{array}$ & $\begin{array}{c}\text { Criado } \\
0 \mathrm{~K} \\
\end{array}$ \\
\hline 1 & $B_{g}$ & 22.9 & 20.7 \\
\hline 2 & $A_{g}$ & 26.6 & 28.9 \\
\hline 3 & $\stackrel{\circ}{A_{g}}$ & 34.3 & 35.7 \\
\hline 4 & $B_{g}$ & 39.9 & 31.2 \\
\hline 5 & $B_{g}^{\circ}$ & 47.4 & 40.2 \\
\hline 6 & $B_{g}$ & 59.7 & 43.6 \\
\hline 7 & $A_{g}$ & 65.7 & 52.9 \\
\hline 8 & $B_{g}^{\circ}$ & 78.3 & 70.7 \\
\hline 9 & $\dot{A_{g}}$ & 91.5 & 81.1 \\
\hline 10 & $A_{g}$ & 94.1 & internal \\
\hline 11 & $B_{g}^{\circ}$ & 100.5 & 98.3 \\
\hline 12 & $\stackrel{\circ}{A_{g}}$ & 107.5 & 118.8 \\
\hline 13 & $B_{g}$ & 108.1 & 114.8 \\
\hline 14 & $A_{u}$ & & 17.2 \\
\hline 15 & $A_{u}$ & & 21.1 \\
\hline 16 & $B_{u}$ & & 23.2 \\
\hline 17 & $B_{u}$ & & 39.6 \\
\hline 18 & $A_{u}$ & & 52.7 \\
\hline 19 & $A_{u}$ & & 93.9 \\
\hline 20 & $B_{u}$ & & 98.2 \\
\hline 21 & $B_{u}$ & & 112.7 \\
\hline 22 & $A_{u}$ & & 121.9 \\
\hline
\end{tabular}

of metastable phases above and below $T_{c}$ or coupling of mode 3 to an infrared (IR) -active mode.

A plausible explanation for the UPRS responses of the low-temperature spectra at $10 \mathrm{~cm}^{-1}$ is that metastable intergrowths of the room-temperature phase are present. The anharmonic response would thus be that of mode 3 associated with a metastable phase trapped as a defect within the stable, low-temperature phase of the crystal. A residual anharmonicity associated with mode 3 is consistent with a thermodynamic first-order phase transition where a metastable phase coexists with a stable phase of the crystal. An optical mode associated with a metastable phase would be expected to exhibit a large anharmon-

TABLE IV. Experimental lattice frequencies $\left(\right.$ in $\mathrm{cm}^{-1}$ ) of the room-temperature orthorhombic phase of phenothiazine.

\begin{tabular}{rcc}
\hline Mode & Symmetry & Nakayama and Ishii \\
\hline 1 & & \\
2 & internal & 22.6 \\
3 & $B_{2 g}$ & 14.7 \\
4 & & \\
5 & $B_{2 g}$ & 39.7 \\
6 & & \\
7 & $A_{g}$ & 60.4 \\
8 & $B_{3 g}$ & 69.0 \\
9 & $B_{2 g}$ & 87.6 \\
10 & $A_{g}$ & 84.9 \\
11 & & \\
12 & $A_{g}$ & 91.1 \\
13 & $B_{1 g}$ & 97.6 \\
\hline \hline
\end{tabular}


icity because of a lack of thermodynamic equilibrium. Responses thought due to metastability were observed for several optical modes of phenothiazine and the modes considered to be metastable are enclosed in brackets in Tables I and II.

If the phase transition of phenothiazine is principally first order, as would be suggested by the formation of metastable phases, the high- and low-temperature phases need not be symmetry related. Therefore, a ferrodistortive, ferroelastic, phase transition would be less likely, though not impossible. There is presently no example of a structural phase transition occurring in an organic crystal where it has been proven that a coupling of the order parameter with a totally symmetric strain produces firstorder behavior. The existence of the metastable phases down to $90 \mathrm{~K}$ is consistent with results of a recent Brillouin experiment. ${ }^{3}$ In that experiment, acoustic phonons were observed to $220 \mathrm{~K}$ where the crystal apparently was still comprised primarily of the original roomtemperature phase.

Theoretically, for a UPRS response two optical modes must be coupling through a mediating externally generated acoustic mode. The coupling optical mode, which may have a frequency quite distinct from the observed Raman band, need not be Raman-active, nor of the same symmetry as the involved Raman mode if coupling is by a nonsymmetric strain. The only requirements are that at least one mode be Raman-active to be observed in the scattering experiment, and that the direct product of the irreducible representation of the symmetries of the coupled optical modes and the strain must contain the totally symmetric representation. Additional information, such as lattice dynamical calculations, knowledge of the eigenvectors of motion, and the anharmonicities of the other modes is needed to determine the nature of the coupled optical mode.

In the lattice dynamical calculations of $\mathrm{Criado}^{20}$ only an IR-active mode $A_{u}$ [assuming a $P 2_{I} / c\left(C_{2 h}\right)$ lowtemperature lattice] at $17 \mathrm{~cm}^{-1}$ displayed any significant variation in frequency under uniaxial pressure. The frequency of this mode, which is librational around an axis near the $b$ axis of the unit cell (monoclinic designation), decreased to $10 \mathrm{~cm}^{-1}$ at $0.74 \mathrm{kbar}$. Raman-active mode 3 was determined to be of $A_{g}$ symmetry and possesses both translational and rotational character with a librational axis also close to $b$. Both these modes were postulated by Criado to be involved in the SPT. At room temperature, it is possible that mode 3 at $15 \mathrm{~cm}^{-1}$ is coupled to the IR-active mode near $17.2 \mathrm{~cm}^{-1}$ since both occur with similar frequency and have similar dynamical character even though they are not of the same symmetry. As mentioned above, the generation of nonsymmetric strains was unavoidable and coupling between phonons of different symmetries is not forbidden for these strains under UPRS selection rules.

The room-temperature UPRS response would then reflect the coupling of these two modes. At $90 \mathrm{~K}$, however, mode 3 shifts to $35 \mathrm{~cm}^{-1}$ and the IR-active mode is also expected to behave similarly since it is calculated to do so under pressure to $10 \mathrm{~cm}^{-1}$. The anharmonicity of the band at $35 \mathrm{~cm}^{-1}$ is $30 \mathrm{~cm}^{-1}$ and that of the band at
$10 \mathrm{~cm}^{-1}$ is $700 \mathrm{~cm}^{-1}$. This coupling is consistent with structural transition of disordered $\operatorname{Pnma}\left(D_{2 h}\right)$ [or disordered $\left.P 2_{I} / c\left(C_{2 h}\right)\right]$ to ordered $P 2_{I} / c\left(C_{2 h}\right)$ with domains.

If the lattice loses a center of inversion through the phase transition, the once Raman-inactive mode now becomes active because inversion symmetry is lost and it may then become observable in the UPRS experiment. For this scenario, the phase transition would be an antiferrodistortive, ferroelastic, phase transition of the type disordered $P n m a\left(D_{2 h}\right)$ or disordered $P 2_{1} / c\left(C_{2 h}\right)$ $\rightarrow P 2_{1}\left(C_{s}\right)$. In either case, to remain consistent with conservation of momentum, the UPRS theory would have to take into account optical-optical, mode coupling with higher-order parameters such as the $S_{35}$ compliance element which has displayed significant anomalous behavior around $T_{c} \cdot{ }^{3}$

It is reasonable that coupling to IR-active modes and metastability are both operant effects in single crystals of phenothiazine. Regardless, the UPRS results support a significant role for the optical modes in the SPT. The normalized, anharmonic responses are larger by at least three orders of magnitude than the largest observed by UPRS for 1,2,4,5-tetrabromobenzene (TBB). ${ }^{13}$ Although phenothiazine is a more compliant crystal than TBB, the degree to which mode 3 displays anharmonicity is significant in light of the Brillouin results.

The coupling constants associated with mode 3 in the frequency range $10-15 \mathrm{~cm}^{-1}$ at room temperature and $35 \mathrm{~cm}^{-1}$ at $90 \mathrm{~K}$ always decrease in magnitude for the various stress configurations: $238 \mathrm{~cm}^{-1}$ to $0 \mathrm{~cm}^{-1}$ for a [001] stress, $85 \mathrm{~cm}^{-1}$ to $-6 \mathrm{~cm}^{-1}$ for a [010] stress and $-26 \mathrm{~cm}^{-1}$ to $-9 \mathrm{~cm}^{-1}$ for a [110] shear. The large reduction of anharmonicity in mode 3 , as the temperature is lowered, suggests that it might be the vibration observed in the NMR experiment of Sircar et al. In that experiment, the freezing out of a particular molecular motion was proposed because of the increase in the rotational second moment $\left(S_{2}\right)$ of a powdered sample as the temperature was lowered. The assignment of the librational axis along a line including the N-S atoms was consistent with their theoretical model. The axial assignment is also in agreement with the lattice dynamical calculations of Criado. ${ }^{20}$ Modes 3 and 14 would have the same librational axes and could couple by an appropriate applied strain.

The UPRS results make the anomalous temperature dependence of the Raman mode 3 more transparent. Above $T_{c}$, mode 3 is coupled to the unstable, IR-active, lattice mode 14. This coupling produces large-amplitude, vibrational motion in mode 3 . As the temperature is decreased, the coupling becomes less pronounced and eventually nonexistent below $T_{c}$ at $90 \mathrm{~K}$. The large anharmonic response at $10 \mathrm{~cm}^{-1}$ in the low-temperature spectra is attributed to either a metastable intergrowth of the room-temperature phase or to the IR-active mode becoming Raman active through the loss of inversion symmetry.

Mode $2\left[22.6 \mathrm{~cm}^{-1}(295 \mathrm{~K}) \rightarrow 26.9 \mathrm{~cm}^{-1}(90 \mathrm{~K})\right]$ has been assigned either as a molecular vibrational mode by Nakayama and Ishii or a lattice mode by Criado. The as- 
sociated Raman band displayed very little temperature or pressure dependence around $T_{c}$ or $P_{c}$ which is consistent with a molecular mode. Mode 2 is sensitive to UPRS, all responses of which display well-defined, derivativelike band shapes. Under all stress configurations, the UPRS responses associated with mode 2 in the high-temperature phases are relatively harmonic (avg. $6 \mathrm{~cm}^{-1}$ ) and they always become more harmonic at low temperature (avg. 1 $\mathrm{cm}^{-1}$ ).

At room temperature there is a large UPRS response at $22 \mathrm{~cm}^{-1}$ and a second derivative band shape is clearly visible at $26 \mathrm{~cm}^{-1}$; the phases of the UPRS responses for the two are $180^{\circ}$ out of phase. At low temperature, there are still two UPRS responses at 22 and $26 \mathrm{~cm}^{-1}$. However, the phases of the responses are the same. Only one conventional Raman band has been observed in the frequency range $20-30 \mathrm{~cm}^{-1}$ at both room temperature and $90 \mathrm{~K}$. This low-temperature band's frequency increased by $5 \mathrm{~cm}^{-1}$, which is consistent with a mode 2 assignment.

Unfortunately, it was not possible to unambiguously assign mode 2 as molecular from the UPRS response because the coupling term does not discriminate between molecular and lattice modes if both are highly anharmonic. In theory, either internal or external optical modes can couple. It is reasonable to assume that molecular modes would be significantly more harmonic than lattice modes. However, such a low frequency is unlikely for a molecular mode for a molecule such as phenothiazine.

Mode 2 may display the same characteristics as mode 3 by coupling to an IR-active mode or being associated with metastability. An explanation for the UPRS results can, nevertheless, be formulated: First, it was determined by Criado that mode 2 is of mixed translationalrotational character with the same symmetry as mode 3 and the same axis of libration as mode 3 and the IRactive mode 14 . Therefore, mode 2 could be coupled to either mode 3 , the IR-active mode 14 , or both, at room temperature. Below $T_{c}$, the coupling becomes less pronounced and either another IR-active mode appears due to the loss of inversion symmetry (from the calculations of Criado two IR-active modes are present in the frequency range $20-30 \mathrm{~cm}^{-1}$ ) or mode 2 is still coupled to the IR-active mode 14 because of a metastable lattice. Thus, the UPRS response in the $20-30 \mathrm{~cm}^{-1}$ frequency range mirrors the response exhibited in the $10-20 \mathrm{~cm}^{-1}$ range.

Mode $4\left[40 \mathrm{~cm}^{-1}(90 \mathrm{~K})\right]$, mode $5\left[39.7 \mathrm{~cm}^{-1}(295\right.$ $\left.\mathrm{K}) \rightarrow 47.4 \mathrm{~cm}^{-1}(90 \mathrm{~K})\right]$, mode $6\left[59.9 \mathrm{~cm}^{-1}(90 \mathrm{~K})\right]$, and mode $7\left[60.4 \mathrm{~cm}^{-1}(295 \mathrm{~K}) \rightarrow 65.7 \mathrm{~cm}^{-1}(90 \mathrm{~K})\right]$ were observed by Nakayama et al., but they did not display any significant UPRS response or were not observable in the experimentally accessible scattering arrangements for these experiments. Mode $8\left[69.0 \mathrm{~cm}^{-1}(295 \mathrm{~K}) \rightarrow 78.8\right.$ $\left.\mathrm{cm}^{-1}(90 \mathrm{~K})\right]$ did exhibit some anharmonicity. The maximum observed was $-81.0 \mathrm{~cm}^{-1}$ for a [110] shear. However, there is not yet enough information to determine the motion or role of this mode in the phase transition.

The coupling constants for modes $9-13$ are shown in Table II. These modes were significantly affected by domain formation, disorder, and domain fluctuation as evidenced by "multiplet" formation. It is also possible that couplings to IR-active modes are affecting their ob- served UPRS responses.

In Figs. 6 and 7 it can be seen that the modes 9-13 all appear as large, broad bands in the $295 \mathrm{~K}$ Raman spectra because of the mixed-symmetry, scattering geometries dictated by the experiment. The individual frequencies could not be determined from the conventional Raman spectra. In the corresponding UPRS spectra, however, individual band shapes can be picked out. Because of the experimental problems alluded to above, it was not always possible to unambiguously assign each mode, much less the sense of the UPRS response. Nevertheless, large anharmonicities are exhibited by some of these modes and new modes not previously recorded are also observed by UPRS.

Two representative curves employing Eq. (10) and used in the determination of coupling constants are shown in Figs. 9 and 10. Note that the room-temperature UPRS response shown in Fig. $7(295 \mathrm{~K})$ displays one broad derivativelike band which suggests one highly anharmonic mode. This experimental data, however, could only be described through Eq. (10) and four, closely spaced or overlapping modes. The congestion, breakthrough modes and subsequent ill-defined band shapes due to "multiplets" prevent an exact duplication of the measured UPRS response. Nevertheless, it is clear that the calculated model band shapes reproduce the gross shape of the UPRS spectra quite well. This analysis demonstrates that, even for a system as complicated as phenothiazine, it is possible to obtain reasonable coupling constants.

Mode 10 [84.0 (295 K) $\rightarrow 94.1(90 \mathrm{~K})]$ was implicated by the lattice dynamics calculations of Criado as the true butterfly mode since this band did not have a calculated counterpart and since $21 \mathrm{~cm}^{-1}$ is too low a frequency for the butterfly mode. The average magnitude of the roomtemperature coupling constants were 16 and $71 \mathrm{~cm}^{-1}$ at room and low temperature, respectively. A molecular mode would be expected to become less anharmonic as

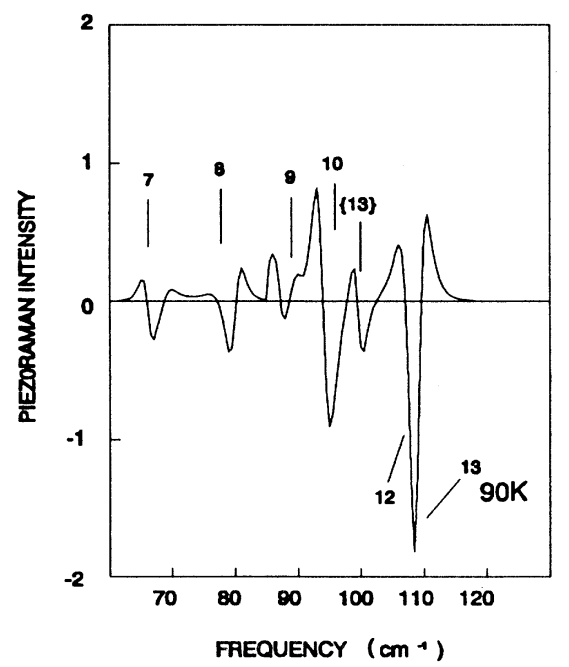

FIG. 9. Calculated UPRS response. Stress: $a b$ shear. Scattering configuration: $a \Varangle b(\{a \Varangle b\} c) a \Varangle b$ at $90 \mathrm{~K}$. 


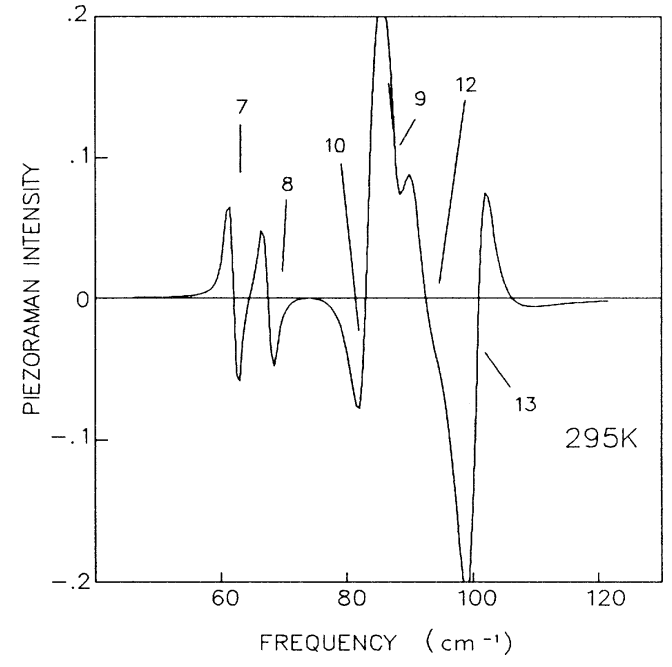

FIG. 10. Calculated UPRS response. Stress: $a$ axis. Scattering configuration: $b(a a) c$ at $295 \mathrm{~K}$.

the temperature is lowered because it would exist in a more harmonic portion of the potential well. Thus, it is probably not a molecular mode.

Mode $9[87.6(295 \mathrm{~K}) \rightarrow 91.5(90 \mathrm{~K})]$ could as easily be assigned as the molecular butterfly mode because it was observed experimentally to have the same symmetry as mode 10. Therefore, the assignment of Criado for modes 9 and 10 could be reversed. The frequency of this mode is closer to the calculated frequency of a vibrational mode in anthracene and displays less temperature dependence.

Nakayama et al. have also implicated mode 12 [91.1 $\left.\mathrm{cm}^{-1}(295 \mathrm{~K}) \rightarrow 107.7 \mathrm{~cm}^{-1}(90 \mathrm{~K})\right]$ in the phase transition. It has been assigned as a pure librational mode of the same symmetry as mode 3 . The average value of the coupling constants observed with mode 12 is $60 \mathrm{~cm}^{-1}$ at room temperature and $45 \mathrm{~cm}^{-1}$ at $90 \mathrm{~K}$.

Nakayama and Ishii observed that the temperature evolution of the linewidth for mode 13 was identical with mode 12. Mode 13 does not, however, display the same temperature and pressure dependence as mode 12 . The average anharmonicities of mode $13,57 \mathrm{~cm}^{-1}$ at room temperature and $40 \mathrm{~cm}^{-1}$ at low temperature, both are very similar to mode 12 .

The UPRS responses of modes 12 and 13 are also curious because at low temperature they apparently become nearly degenerate. The conventional Raman band associated with these two modes appears as a single narrow peak; however, the UPRS response can only be explained as two bands. The UPRS band shape in the frequency range $107-110 \mathrm{~cm}^{-1}$ in Fig. 7 (90 K spectra) looks like an inverted peak and could only be fit with two, relatively anharmonic, modes (see Fig. 9).

A large anharmonic response was observed at 100 $\mathrm{cm}^{-1}$ at room temperature and is associated with mode $11\left[100.0 \mathrm{~cm}^{-1}(90 \mathrm{~K})\right]$. The average, room-temperature value is $550 \mathrm{~cm}^{-1}$. Even the low-temperature coupling constants of this mode are large, being $81 \mathrm{~cm}^{-1}$ on average.
Three other modes give significant UPRS responses but were not recorded by Nakayama and Ishii. They do appear in the lattice dynamic calculations of Criado. Of the three, $73,112.5$, and $118 \mathrm{~cm}^{-1}$, the band with a frequency at $73 \mathrm{~cm}^{-1}$ is the most anharmonic with an absolute anharmonicity of $424 \mathrm{~cm}^{-1}$.

\section{CONCLUSION}

The UPRS experiment has shown that many of the optical modes of phenothiazine are significantly anharmonic above and below $T_{c}$. Modes 2, 3, 10, 11, 12, 13, and the mode with a frequency at $73 \mathrm{~cm} \mathrm{~cm}^{-1}$ (at $100 \mathrm{~K}$ ) displayed the largest responses. Mode 3 exhibits the most striking anharmonic behavior with change in temperature. Instabilities in this mode or the coupled mode are directly related to, or are the primary order parameter for, the phase transition. The damping of librational motion revealed by NMR experiments mirrors the observed UPRS responses and is probably associated with mode 3 . In this study an unambiguous assignment of the molecular butterfly mode could not be made based on the UPRS responses because of the anisotropy of the lattice and the inability to generate a pure strain.

An entirely different picture than heretofore postulated can be drawn for the phase transition in phenothiazine. If the UPRS responses are the result of a metastable intergrowth, i.e., the presence of the orthorhombic, roomtemperature structure trapped as a defect in the lowtemperature phase, the transition must be first order. In this case, Landau or "soft mode" theory does not apply, nor do the room- and low-temperature structures have to be related by symmetry. Previous Brillouin scattering data support the presence of domains above and below $T_{c}$ with domain boundaries blurred by disorder. The phase transition could thus be described as a molecular ordering within static, orthorhombic-domain superstructures. Instabilities associated with mode 3 would then be driving the structural transformation.

Although many of the modes between 10 and 100 $\mathrm{cm}^{-1}$ exhibited possible metastable effects, above and below $T_{c}$, it is not possible to rule out coupling to unstable IR-active modes. In view of the anharmonic behavior observed between 10 and $20 \mathrm{~cm}^{1}$, two alternative mechanisms would emerge for the phase transition. As the temperature is increased in the low-temperature phase through $T_{c}$, optical modes 3 and 14 become more anharmonically coupled due to an unstable element of the compliance tensor $S_{35}$. The coupling would produce largeamplitude molecular-librational motions in one of the modes, probably mode 14 . When the temperature reaches $T_{c}$, the librational instabilities make the molecules either distribute between two disordered positions or undergo large anharmonic vibrations about an unstable position. The second mechanism is similar to the first except that modes 3 and 14 are coupled to a $\hat{q} \neq 0$ acoustic phonon.

The first mechanism is not inconsistent with an improper ferroic change in lattice structure of a disordered, orthorhombic, Pnma $\left(D_{2 h}\right)$ lattice to an ordered, 
$P 2_{1} / c\left(C_{2 h}\right)$ lattice with domains. It is also compatible with a simple order-disorder transition if the roomtemperature structure is actually a polysynthetically twinned monoclinic crystal as suggested by van der Waal and Feil. The second mechanism results in loss of inversion symmetry in the lattice, leading possibly to an ordered $P 2_{1}\left(C_{s}\right)$ lattice structure with domains (a known polymorph for phenothiazine). A weak pyroelectric effect has been observed for phenothiazine at low temperature ${ }^{3,15}$

Metastability and IR coupling are not mutually exclusive and both effects may be occurring. Further refinements of the room- and low-temperature lattice structure would be considerably helpful. Future work should address the experimental temperature dependence of the IR-active modes, especially mode 14 , as well as the role of $\hat{q} \neq 0$ acoustic modes.

The study has demonstrated the efficacy of using uniaxial, piezomodulated Raman spectroscopy in the study of phase transitions. The application of UPRS to phenothiazine has proven to be effective in locating structure and phase behavior that has been otherwise undetected by conventional Raman scattering. In particular, the UPRS has permitted quantitative evaluation of the degree of anharmonicity for lattice modes involved in the phase transition.
"Present address: Harris Laboratories, 624 Peach Street, Lincoln, NE 68501.

†Author to whom correspondence should be addressed.

${ }^{1}$ H. Nakayama, K. Ishii, E. Chijiwa, M. Wada, and A. Sawada, Solid State Commun. 55, 59 (1985).

${ }^{2}$ C. Ecolivet, M. Sanquer, K. Ishii, and H. Nakayama, Phys. Rev. B 44, 4185 (1991).

${ }^{3}$ J. Sartwell and C. J. Eckhardt, Phys. Rev. B (to be published).

${ }^{4} \mathrm{H}$. Nakayama and K. Ishii, Chem. Phys. 114, 431 (1987).

${ }^{5}$ G. Araki, M. Mukai, H. Nakayama, and K. Ishii, Bull. Chem. Soc. Jpn. 46, 897 (1990).

${ }^{6}$ R. Sircar, S. C. Mishra, and R. C. Gupta, Indian J. Pure Appl. Phys. 18, 757 (1980).

${ }^{7}$ S. Ishimaru, N. Nakamuro, and H. Chihara, J. Phys. Soc. Jpn. 60, 2323 (1991).

${ }^{8}$ H. Nakayama, K. Ishii, and A. Sawada, Solid State Commun. 67, 179 (1988).

${ }^{9}$ M. Born and K. Huang, Dynamical Theory of Crystal Lattices
(Clarendon, Oxford, 1954).

${ }^{10}$ T. Luty and C. J. Eckhardt, J. Chem. Phys. 92, 2214 (1985).

${ }^{11}$ K. White and C. J. Eckhardt, Phys. Rev. Lett. 59, 574 (1987).

${ }^{12}$ K. White, Ph.D. Dissertation, University of Nebraska, 1987.

${ }^{13}$ K. White and C. J. Eckhardt, J. Chem. Phys. 92, 2214 (1990).

${ }^{14}$ E. B. Wilson, J. C. Decius, and P. C. Cross, Molecular Vibrations (McGraw-Hill, New York, 1955).

${ }^{15}$ B. W. van de Waal and D. Feil, Acta Cryst. B 33, 314 (1977).

${ }^{16}$ R. D. Stigler, Ph.D. Dissertation, Universität Stuttgart, 1988; J. J. Stezowski (private communication).

${ }^{17}$ M. C. Tobin, in Laser Raman Spectroscopy, Chemical Analysis Vol. 35, edited by P. J. Elving and I. M. Kolthoff (WileyInterscience, New York, 1971).

${ }^{18}$ M. Morrow and C. J. Eckhardt (unpublished); M. Morrow, Ph.D. dissertation, University of Nebraska, 1988.

${ }^{19}$ S. P. S. Porto, J. A. Giordmaine, and T. C. Damen, Phys. Rev. 147, 608 (1966).

${ }^{20}$ A. Criado, Solid State Commun. 76, 61 (1990). 